\title{
DEVELOPMENT OF A SAFETY INDUSTRY 4.0 ProduCTION ENVIRONMENT
}

\author{
Maximilian Papa, David Kaselautzke, Thomas Radinger, Kemajl Stuja
}
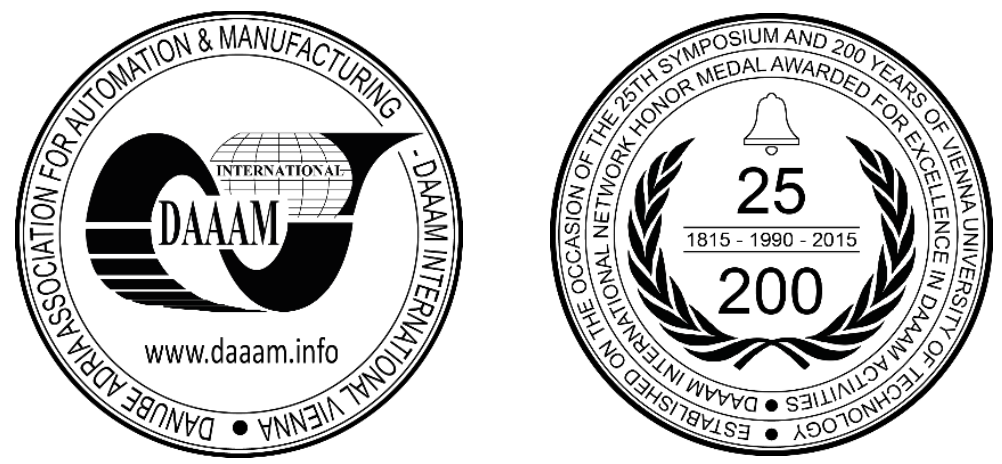

This Publication has to be referred as: Papa, M[aximilian]; Kaselautzke, D[avid]; Radinger, T[homas] \& Stuja, K[emajl] (2017). Development of a Safety Industry 4.0 Production Environment, Proceedings of the 28th DAAAM International Symposium, pp.0981-0987, B. Katalinic (Ed.), Published by DAAAM International, ISBN 978-3-90273411-2, ISSN 1726-9679, Vienna, Austria

DOI: $10.2507 / 28$ th.daaam.proceedings.136

\begin{abstract}
In modern industrial environments, the usage of autonomous mobile robots is very common. Building up the mobile robot's prerequisites not only financial resources but interdisciplinary knowhow too. Therefore, these preconditions barriers small and medium-sized companies from the up to date industrial concepts. In order, to support small and medium-sized companies this paper shows, how to design and implement mobile robot systems capable to work safety into complex industrial 4.0 environment.

This research task can be accomplished in three steps. The first step deals with the conceptualization and creation of a test environment inspired by a small and medium-sized environment. The difficulty is to build a new suitable test factory, which fits in an office room. In the second step, a simple work station for different test cases were conceptualized. The third step deals with the creation of different mobile robots. Because of the limited space the mobile robots in this work were not bigger than $20 \times 20 \times 20 \mathrm{~cm}$.

A concept for a test industry 4.0 test factory with simple work stations was created. Different miniature mobile robots were bought and tested, whereby different risks of the standards were researched. For the next steps, an implementation and guideline of a solution in a small- or medium-sized company will be tested and written.
\end{abstract}

Keywords: safety; mobile robot; SIP4.0; ROS

\section{Introduction}

Nowadays the industry gets more often confronted with the terms of "industry 4.0 " or "internet of things". These terms stand for an intelligent cooperation of devices in a factory which are communicating with each other. The great advantage for a company will be a flexible production line and that the products can be produced individual for the respective customer. [1] Therefor the change to industry 4.0 comes also with a competitive advantage against former industry companies. But there is a big difference between big-sized companies to small- and medium-sized companies. Namely the smaller companies normally don't have as much money to invest for this change. [2]

The goal of this paper will be to create and test a cost-efficient mobile robotic solution in the industry 4.0 environment. Mobile robots are used for the material transportation between different work stations and machines and in an industry 
4.0 environment they have act full autonomy. That means to avoid obstacles and plan their path to the right work station. This goals obviously must confirm with the actual standards and guidelines. [3]

Further the safety and security are big parts of the Industry 4.0 and is often called as "SIP4.0 - Safety \& Security in Industry 4.0 environments". Security is used for the secure connection and communication between the different devices. But the security will not be processed in this paper, instead the safety of the mobile robots in an industry 4.0 environment will be dealt with. Therefor different problems must be solved. First the safety aspects should be thought off. The mobile robot must be designed and programmed so it won't harm people or other machines under no circumstances. If it is not possible, the danger must be set to the lowest possible way. [4]

For these reasons a suitable miniature industry 4.0 test factory must be build. To work within an office room with more than one robot and different stations at the same time, the whole test factory within the stations and mobile robots must be very small. Furthermore, the mobile robots were chosen with a size not larger than 20x20x20cm for an optimal test place.

Another goal in this work is, that the whole hardware- and software-solution could be easily transformed to the real and bigger mobile robots, after the successful completion of this work. Through the limitation in financial resources some cost-efficient solutions should be created, because these solutions are very interesting for the small- and medium-sized companies. Once the creation of the test factory with different stations and at least two mobiles robots is done, it should be tested for a SIP4.0 Standard.

Obvious all build robots must be built within the newest safety standards and the state of the art robot controller. Additionally, all stations and mobile robots should have prepared a way for the network communication. This will be needed, if the mobile robot should be requested by the station, whereby the mobile robot must navigate locally to the right station through moveable and fixed obstacles. To avoid a harm from the mobile robot for humans or other machines, all standards will be influenced in this context.

\section{State of the art and research}

The first step for the mobile robots in the safety industrial environment is to design them properly. Per definition of ISO 13482:2014 (Robots and robotic devices - Safety requirements for personal care robots) [5] a mobile robot is a personal care robot. The ISO 13842:2014 shows that a risk assessment for a safety concept for a mobile robot in an industrial environment is necessary. The exact definition and concept of the risk assessment is shown in ISO 12100 (Safety of machinery -- General principles for design -- Risk assessment and risk reduction) [6], which contains the provision of a risk assessment and a risk analysis on basis of possible danger identifications.

For a risk assessment it must be clear, if a risk is acceptable or not. Therefore, in the first place an inherent construction of the mobile robot should be made. If this isn't practicable it is necessary to implement protective measures at the mobile robot. For last instance, if there is any dangerous possibility left caused due to the mobile robot, it is required to write user information's of them into the user manual. In other place, for dangerous risks which cannot get solved it is necessary to have warning signs directly mounted on the mobile robot or at the factory which can be directly recognized from every human.

Some danger identifications for a risk assessment are listed in chapter 4.2 or in Annex A in ISO:13482. However, it should be considered that this list is not a complete list of all risks with a mobile robot. Thus, for a risk assessment it is important to list every single risk which stands in connection with the mobile robot and should be supplemented for every other application.

After all possibilities for an inherent construction and protective measures for the unsolved risk were made a residual risk of the mobile robot must be calculated. However, an acceptable risk of the mobile robot should be audited. In ISO:13842 it is recommended to setting up a risk assessment as individual cases. Thereby an acceptable risk can be evaluated. For example, if a mobile robot is allowed to enter safety-relevant obstacles or not.

For a state-of-the-art solution regarded to the robot controller the robot operation system (ROS) will be used. ROS is an open-source framework especially design for the robotic use. Many programmers around the world are using this framework and extend the content with their own programs. This concept allows an easy and fast way to program a robot, without programming each function from the start on the own. [7]

\section{Methods}

In this chapter are the different concepts listed for the planed test factory in the office room. When this is successful and has proven, it can get transformed for a small or medium-sized company.

\subsection{Factory concepts:}

Three different test factory concepts have been created. In the first concept an ideal factory with perfect pasted lines on the floor was thought off. The mobile robot would use this lines for the navigation through the factory from station to station. The symbolic sketch of this concept can be seen in Fig. 1. 


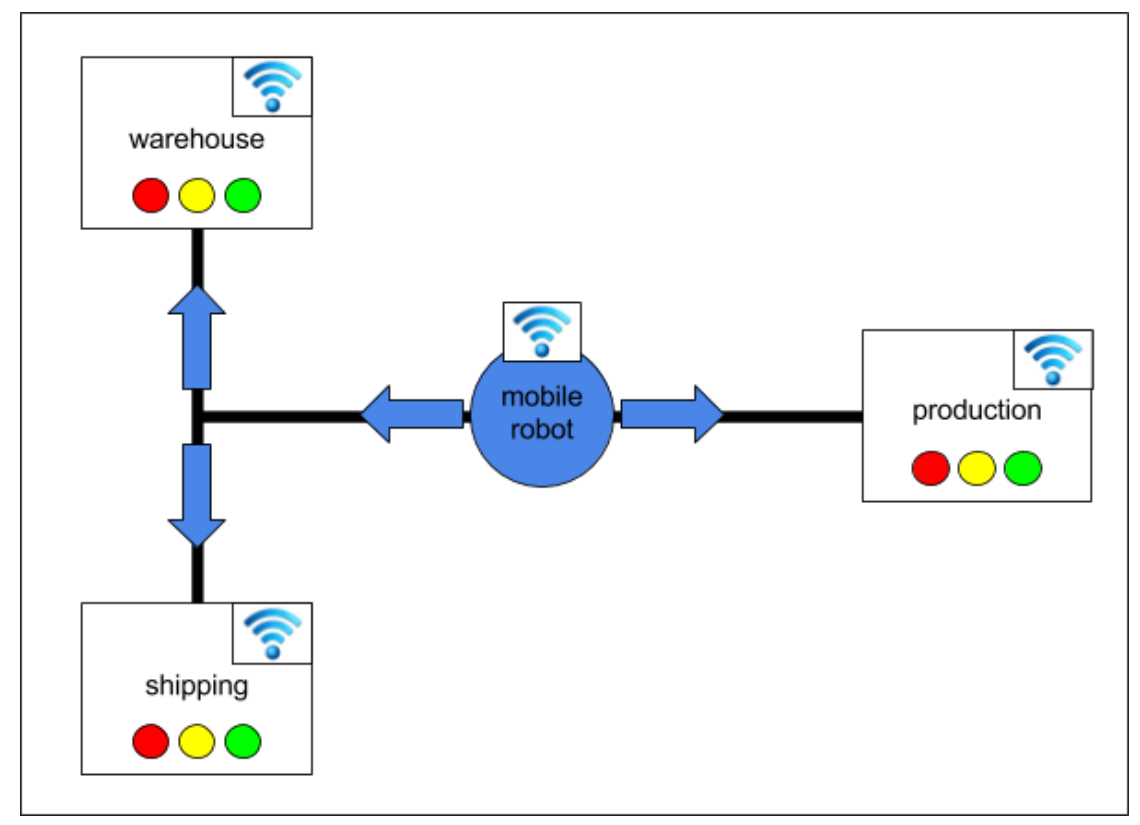

Fig. 1. Concept of an SIP4.0 factory with pasted lines

In an actual small- and medium-sized-factory it is nearly impossible to work with this kind of navigation. Possible errors in a small- and medium-sized company with pasted lines would be strong wear or pollution of the lines. Also, a perfect contrast between the lines and the ground is rare and if there is a high contrast, it is possible that the sun irritates the line following sensors of the robot. Additionally, sometimes people or machines would cross the lines of the robot, which have to avoid a collision.

Because of these reasons a second concept for the test factory was created. In this concept a two-dimensional plan of the factory would be generated. The factory would be divided in rectangles of the same height and width, preferably in the size of the stations or the mobile robot. This plan would be saved into the mobile robot and would be represented there through an array. With this array the mobile robot knows the factory structure and could plan the path global through the factory. For this path planning an algorithm named A* would be very common. [8]

On the other hand, for the local navigation sensors like a gyroscope, odometry or infrared could be used. The infrared would be used to detect obstacles, which are not recorded in the global array plan and for a communication with other mobile robots and working stations. Therefore, the work stations would have an infrared transmitter, so the robot have a redundancy to know which station is sending infrared data and knowing also, due to that where his position in the factory is. But a big disadvantage of this concept is the weakness in dynamic factory structures. Because each time the factory arrangement would change, the whole plan or array must be updated and saved into the robot again. The symbolic sketch of this second concept is shown on the Fig. 2.

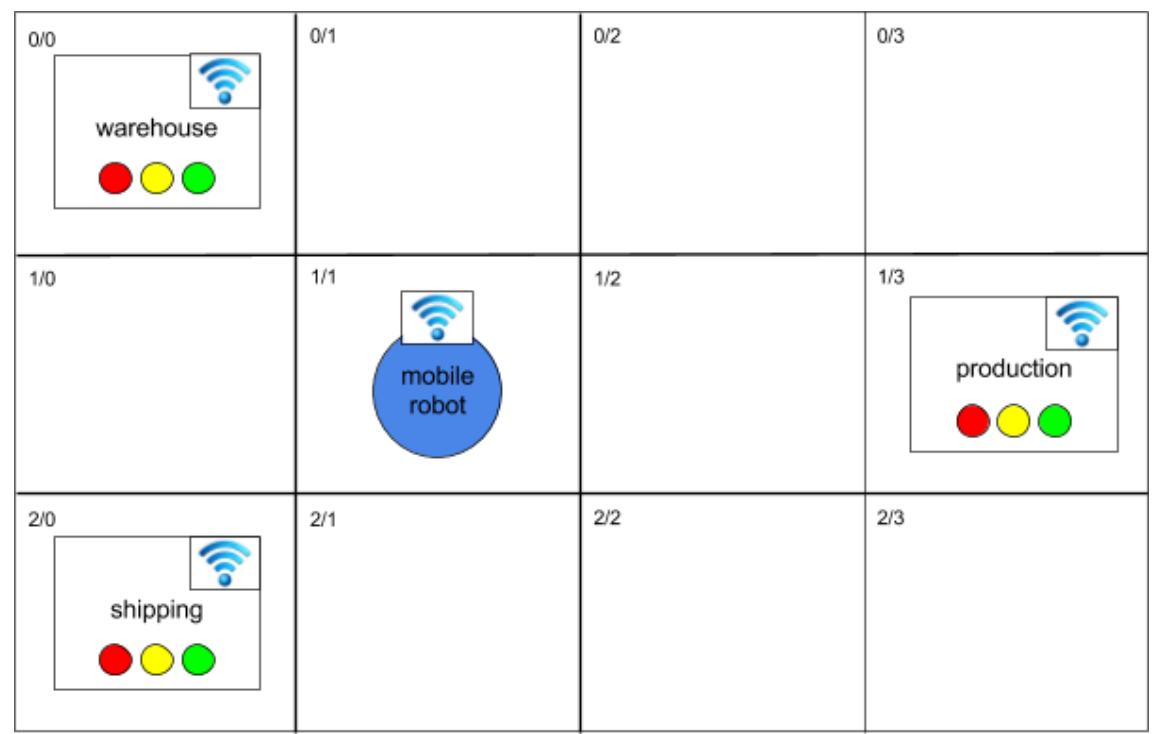

Fig. 2. Concept of a SIP4.0 factory with a two-dimensional plan 
The second concept has how already mentioned a big disadvantage with big and variable stations which has a dynamic factory structure. However, the plan creation must be perfect and this is very exhausting for the user when it comes to rebuilding it. Therefore, a third concept for small- and medium-sized companies were created. These factories of this companies are in most cases chaotically formed because they have been growing over time. Thus, these factories didn't have perfect structed machines and working stations. In addition, obstacles like humans or lift trucks can get in the way of mobile robots.

Because of these points in this third concept all machines (stations) can communicate with the mobile robots which calculates its way to the stations completely dynamical with an additional local obstacles avoidance. With infrared and ultrasonic sensors, the local obstacle avoidance will be made. In addition, the infrared signals will be used for a station recognition like in the second concept. Optional the mobile robot could use a camera or a laser to generate a dynamic plan of the factory. Through appropriate placement of the sensors the robot can identify the height of the obstacles and can drive beneath tables or chairs for example. [3]. Also for this third concept a symbolic sketch was included as Fig. 3.

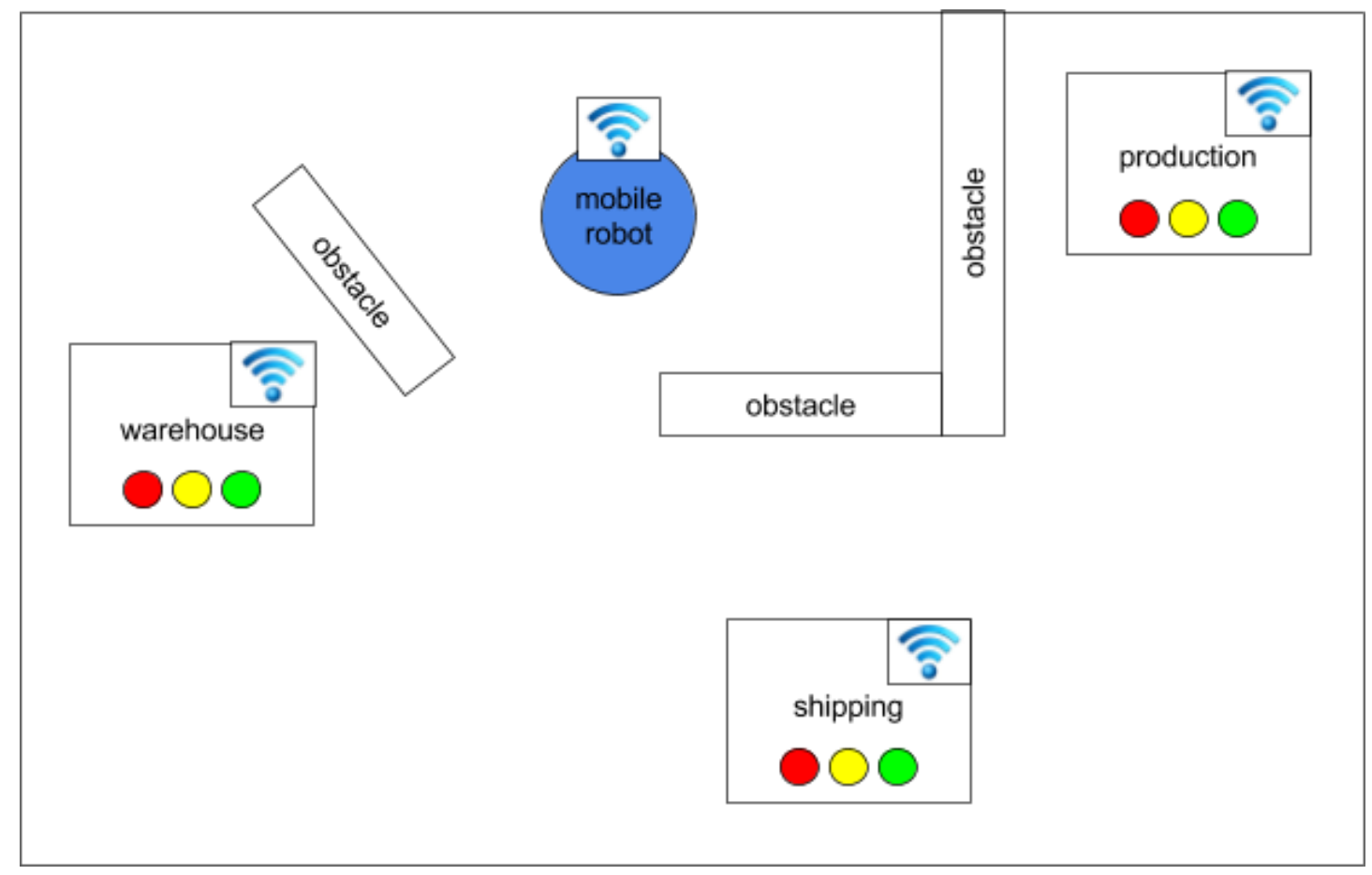

Fig. 3. Concept of a chaotically formed factory

This concept would be the best fitting concept to build in every small- and medium-sized company. With a laser sensor the mobile robot could generate a map of the factory for the self-localization in a dynamically created global map. In addition to that, the mobile robot could get information's where the allowed safety areas to drive though are. This should be used as redundancy for the safety driving along safety areas in the factory.

\subsection{Station concepts:}

After the concept of the own test factory was clear, the stations, which represent the machines in this test factory, and the mobile robots were conceptualized. First it was considered which states are necessary to display different test cases. For the communication between the control system and every station a wireless network access is needed. Therefore, a Raspberry Pi (RPi) will be used. However, two different Raspberry Pi's got analysed in greater detail [9]:

\begin{tabular}{|c|c|c|}
\hline & Raspberry Pi 3 & Raspberry Pi Zero W \\
\hline Size & $85,6 \mathrm{~mm} \times 56 \mathrm{~mm}$ & $65 \mathrm{~mm} \times 30 \mathrm{~mm}$ \\
\hline CPU cores & 4 & 1 \\
\hline CPU frequency & $4 \times 1200 \mathrm{MHz}$ & $512 \mathrm{MB}$ \\
\hline RAM & $1024 \mathrm{MB}$ & Yes \\
\hline WLAN & Yes & $1000 \mathrm{MH}$ \\
\hline
\end{tabular}

Table 1. Comparison of Raspberry Pi 3/Zero W 
The Raspberry Pi Zero W and the Raspberry Pi 3 provide both a WLAN communication. But in combination with ROS the RPi Zero W would be too weak because of its lower specifications like the CPU und RAM. [10]

Thus, the Raspberry Pi 3 was chosen. The RPi was also chosen because it is possible to run Linux with ROS on it. If a ROS connection isn't required for the mobile robot, it is possible to use a combination of an Arduino and an WLAN module. The best module with the best value of money is the ESP8266. With this module it is possible to provide a WLAN connection to communicate with the control system of the test factory.

For the stations it is not necessary to supply them with big tasks in first place. To begin with different states of the stations will get visualized with LEDs. Therefore, every station has three LEDs for the state recognition. It can visualise that the station is free, is working or is ready to collect. Further an infrared transmitter is planned for a local communication between station and mobile robot. And of course, also a limit switch will be installed, to recognise the delivery of the mobile robot.

\subsection{Mobile robot concepts:}

As mentioned before it is necessary that at least more than two mobile robots are working simultaneous at the test factory. Because the test factory will be built in an office room with limited space, the robots should need lesser space than 20x20x20cm, Furthermore, the dodge scenarios and the communication could only be tested with more than two mobile robots. To build an own mobile robot the

Table 2 lists important components which were researched and needed for a simple tiny mobile robot.

\begin{tabular}{|c|c|c|c|}
\hline Component & Costs per unit [€] & Units & Costs [€] \\
\hline Gear motors & 4,6 & 1 & 1,6 \\
\hline Encoder & 14,65 & 1 & 3,5 \\
\hline Driving wheel & 3,5 & 1 & 4,05 \\
\hline Support wheel & 4,05 & 2 & 21,2 \\
\hline Infrared Sensor & 10,6 & 1 & 19,95 \\
\hline Arduino & 19,95 & 1 & 18,99 \\
\hline Raspberry Pi & 18,99 & 1 & 1,49 \\
\hline SD card & 11,49 & 1 & 1,99 \\
\hline Ultrasonic sensor & 10,99 & 1 & 0,51 \\
\hline Battery holder & 1,99 & 1 & 18,99 \\
\hline Battery clip & 0,51 & 1 & 3,59 \\
\hline Accumulator & 18,99 & 1 & 0,26 \\
\hline Motor driver & 3,59 & 1 & 0,26 \\
\hline IC socket & 0,26 & 1 & 1,09 \\
\hline Voltage regulator & 0,26 & 1 & \\
\hline Heat sink & 1,09 & & 1 \\
\hline
\end{tabular}

Table 2. Components for an own tiny mobile robot

The total costs for the own built mobile robot would be $136.11 €$ without any circuit boards or the whole assembly. Simultaneous to the research of the components some existing complete miniature mobile robots were compared. The research showed, that the existing mobile robots where much cheaper than self-built one. The self-built one would be more flexible, but in order to the limited time and budget some on the market existing tiny mobile robots where bought based on the comparison in

Table 3:

\begin{tabular}{|c|c|c|}
\hline $\begin{array}{c}\text { Mobile } \\
\text { Robot }\end{array}$ & Advantages & Disadvantages \\
\hline $\begin{array}{l}\text { AREXX } \\
\text { AAR } 04\end{array}$ & $\begin{array}{l}\text { + Integrated Arduino } \\
+ \text { Very cheap } \\
\text { + Optical Line following } \\
\text { + Plug and play } \\
\text { + Odometry sensors }\end{array}$ & $\begin{array}{l}\text { - Rectangle shape } \\
\text { - No Infrared or ultrasonic sensor } \\
\text { available }\end{array}$ \\
\hline $\begin{array}{l}\text { Elegoo } \\
\text { UNO }\end{array}$ & $\begin{array}{l}\text { + External Arduino programming } \\
\text { + Ultrasonic sensor } \\
\text { + Line following } \\
\text { + Infrared and Bluetooth remote controlling }\end{array}$ & $\begin{array}{l}\text { - No infrared sensors } \\
\text { - Oval shape }\end{array}$ \\
\hline
\end{tabular}




\begin{tabular}{|c|c|c|}
\hline FRANZIS & $\begin{array}{l}\text { + Integrated Arduino } \\
\text { + Big round platform for expansions } \\
\text { + Line following }\end{array}$ & $\begin{array}{l}\text { - Discontinued by Arduino } \\
\text { - No ultrasonic sensors }\end{array}$ \\
\hline $\begin{array}{l}\text { NIBO } \\
\text { Burger }\end{array}$ & $\begin{array}{l}\text { + Integrated Arduino } \\
+ \text { Line following, Robot following, Obstacle avoidance, } \\
\text { Colour detection } \\
+4 \text { infrared sensors, } 2 \text { infrared wheel encoders } \\
\text { + } 4 \text { programable LEDs on top of the mobile robot } \\
+ \text { Documentation and libraries } \\
+\mathrm{I}^{2} \mathrm{C}, \text { UART, SPI interface for Raspberry Pi } \\
+ \text { Expansions possible on top of the robot }\end{array}$ & - No ultrasonic sensors \\
\hline Variobot & $\begin{array}{l}\text { + Line and light following } \\
\text { + Interaction with obstacles and other robots } \\
\text { + Obstacle avoidance (due to patented sensors) }\end{array}$ & - No programming possible \\
\hline $\begin{array}{l}\text { ZUMO } \\
\text { Robot }\end{array}$ & $\begin{array}{l}\text { + Integrated 32U4 microcontroller } \\
\text { + Line following, Robot following, Obstacle avoidance } \\
+4 \text { infrared sensors, Wheel encoders } \\
\text { + Documentation and libraries } \\
\text { + Expansions possible on top of the robot }\end{array}$ & - No ultrasonic sensors \\
\hline
\end{tabular}

Table 3. Comparison of different mobile robots

The different advantages and disadvantages of each existing mobile robot were compared and as a result some NIBO Burger and ZUMO Robots were bought. Both mobile robots offer many useful functions as line tracking or obstacle avoidance. The NIBO Burger is the more cost-efficient solution, but it uses a plastic gearbox instead of a metal one like the ZUMO robot. Also, both bought mobile robot systems are working with an Arduino processor, which unfortunately cannot be used directly with ROS. A Raspberry Pi 3 with the Ubuntu Mate operating system will be needed, where the ROS package could be installed for the robot controlling. With the combination of the Arduino processor and the Raspberry Pi with the ROS package some basic question about safety and the communication in the industry 4.0 environment can be solved.

If a Raspberry Pi will be used, an external power supply will be needed. A power bank would offer the needed portability and the output current of minimum two amperes. All used mobile robots are needing modifications for this work.

\section{Results}

To compare the different mobile robot solutions and the possible needed improvements the ZUMO Robot and the NIBO Burger were tested in some load tests.

The ZUMO Robot was supplied a $2700 \mathrm{mAh}$ accumulator, where the robot drove in an obstacle avoidance program full automatically. Under full motor speed the ZUMO mobile robot was capable to drive over 3 hours and 45 minutes. This long test drive was very overwhelming, but even if longer running time is required, the power supply can be extended by a power bank. Further, the robot hit often an obstacle, because the infrared sensors are not covering the whole robot body. The edge, the middle front and the top obstacle avoidance of the robot could be improved through more or better placed infrared and additional ultrasonic sensors. Therefor the sensors must be placed at the top of the robot so it can recognize, if the robot can drive beneath obstacles or not.

At the research of the standards some risk analyses for the mobile robot were done and listed in the next few sentences:

- The mobile robot may not squeeze persons or machines in a crash. To avoid this kind of injuries, the mobile robot must ensure different safety precautions, like bumpers.

- The gears are dangerous if they are reachable, in order they must be hidden under cases. Also, other harmful mechanical components must be protected by a case.

- The mobile robot must ensure precautions against unintentional contact with the power supplies. Again, a case around the dangerous components would help to prevent any harm.

- All outer sides of cases and the mobile robot should not be maintaining sharp edges, where persons or machines could injury in a collision.

- The mobile robot may not move spontaneous over a big distance after turning it on or after a warm restart.

- In the end may all risks though emission like noise emission, risks through vibrations or extreme temperature be prevented.

All above written risks must be ensured and have precautions to build a standardized mobile robot. But these are only a few very important risks for the final mobile robot concept which will have a new and more detailed risk analysis. This will be made for the finished test factory and is not already necessary along the standpoint of this project. 


\section{Discussion}

This project has just started and is in the initial phase. In first place the research began about the topic SIP 4.0. Afterwards the goal was to develop concepts for a mobile robot for companies in small- and medium-sized companies. Due to that it was decided to build an own test factory in a miniature design in an office room to have infinite possibilities for expanding and designing a mobile robot and working stations.

In the next step the mobile robots, which were already tested, will get expanded with Raspberry Pi's for a communication between each mobile robot and the working station. Additionally, the robots will get ultrasonic sensors and replaced infrared sensor for a better obstacle avoidance. However, the next step is to build the working stations and equip them with Raspberry Pi's or ESP8266 for an WLAN communication in the test factory with the mobile robots.

Another improvement for the mobile robots would be the extension with a camera. Especially for the concept of a chaotically formed factory would the additional camera navigation bring benefits. For example, an improved obstacle avoidance through visual detection or for a tele-operated emergency function. Furthermore, the mobile robot needs a way to localize in an industry 4.0 indoor application, because GPS is not working there. Through the camera special natural landmarks could be detected. [11] And with the detected landmarks the path of the mobile robot could get planned. [12]

When this is done, the project goes into the next step which means to transform this test factory to a fully SIP 4.0 factory. Therefor the topics safety and security of the whole factory will get updated to the state of the art. If this is working together the project will begin to expand with bigger mobile robots, which will get equipped with the safety and security standards of the miniature mobile robots. Therefore, a risk assessment as it stands in the ISO 13482 will created.

The final goal will be to have a mobile robot for a small- or medium-sized company which get tested for an autonomous system which brings parts from Machine A to B. Therefor the mobile robot may get equipped with a robotic arm to grab parts from the machines and delivers them. Based on these results a guideline for the usage of cost-efficient mobile robots for companies in an industry 4.0 environment will be created.

This miniature test factory will help for the research of an optimal SIP4.0 production environment. With the created and tested smaller mobile robots all important questions, methods and standards were prepared and can easily adapted on bigger mobile robots. For different test cases the test factory and their stations, built in this work, can be used.

\section{Acknowledgments}

We would like to thank the "MA23" and also the UAS Technikum Wien for the opportunity to work on this paper and its very interesting topic. This accomplishment would not have been possible without them and therefor a big thank you.

\section{References}

[1] Stojkić, Ž.; Veža, I. \& Bošnjak, I. (2016). „A concept of information system implementation (CRM and ERP) within Industry 4.0“, Proceedings of the 26th DAAAM International Symposium, pp.0912-0919, Vienna, Austria.

[2] Bischoff, J.; Taphorn, C.; Wolter, D.; Braun, N.; Fellbaum, M.; Goloverov, A.; Ludwig, S.; Hegmanns, T.; Prasse, C.; Henke, M.; Hompel, M.; Döbbeler, F.; Fuss, E.; Kirsch, C.; Mättig, B.; Braun, S.; Guth, M.; Kaspers M. \& Scheffler, D. (2015). „Erschließen der Potenziale der Anwendung von ,Industrie 4.0` im Mittelstand“, Mülheim an der Ruhr: agiplan $\mathrm{GmbH}$.

[3] Siegwart, I. N. R. (2004). „Introduction to Autonomous Mobile Robots”, MIT Press.

[4] Markis, A.; Montenegro, H.; Neuhold, M.; Oberweger, A.; Schlosser, C.; Schwald, C.; Sihn, W.; Ranz, F.; Edtmayr, T.; Hold P. \& Reisinger, G. (2016). „Sicherheit in der Mensch-Roboter-Kollaboration“, TÜV AUSTRIA Holding AG \& Fraunhofer Austria Research GmbH, Wien.

[5] ISO 13482:2014, (2014). „Robots and robotic devices -- Safety requirements for personal care robots”.

[6] DIN EN ISO 12100:2011-03, (2011). „Safety of machinery - General principles for design - Risk assessment and risk reduction".

[7] http://www.ros.org, (2017). „Robot Operating System”, Accessed on: 2017-09-25.

[8] Hart, P. E.; Nilsson, N. J. \& Raphael, B. (1968). „A Formal Basis for the Heuristic Determination of Minimum Cost Paths“, IEEE Transactions on Systems Science and Cybernetics, Bd. 4, Nr. 2, pp. 100-107.

[9] https://www.datenreise.de/raspberry-pi-unterschiede-zwischen-den-modellen/, (2017). „Raspberry Pi Modellvergleich“, Accessed on: 2017-09-15.

[10] https://kofler.info/ubuntu-mate-16-04-raspberry-pi/, (2016). „Ubuntu Mate 16.04 für den Raspberry Pi”, Accessed on: 2017-09-12

[11] Smirnova, E.; Stepanov, D. \& Goryunov, V. (2016). „A technique of natural visual landmarks detection“, Proceedings of the 26th DAAAM International Symposium, pp.0905-0911, Vienna, Austria.

[12] Katsurin, A. (2016). „Planning trajectory of the mobile robot with a camera“, Proceedings of the 26th DAAAM International Symposium, pp.0407-0416, Vienna, Austria. 